\title{
Activités
}

5-1 | avril 2008

Varia

\section{Suivre à la trace l'activité de deux co-acteurs}

Le cas d'une rédaction conjointe médiée par un artefact numérique

Tracking two co-users' activity: The case of a joint writing situation mediated by a numerical artefact

Seguir de cerca la actividad de dos co-autores : el caso de redacción conjunta mediada por un artefacto numérico

\section{Magali Beldame}

\section{OpenEdition}

Journals

Édition électronique

URL : http://journals.openedition.org/activites/1926

DOI : 10.4000/activites. 1926

ISSN : 1765-2723

Éditeur

ARPACT - Association Recherches et Pratiques sur les ACTivités

\section{Référence électronique}

Magali Beldame, «Suivre à la trace l'activité de deux co-acteurs », Activités [En ligne], 5-1 | avril 2008,

mis en ligne le 15 avril 2008, consulté le 19 avril 2019. URL : http://journals.openedition.org/ activites/1926 ; DOI : 10.4000/activites.1926

\section{(c) (i) (3) $\Theta$}

Activités est mis à disposition selon les termes de la licence Creative Commons Attribution - Pas d'Utilisation Commerciale - Pas de Modification 4.0 International. 


\title{
Suivre à la trace l'activité de deux co-acteurs : \\ Le cas d'une rédaction conjointe médiée par un artefact numérique
}

\author{
Magali Beldame \\ mbeldame@liris.cnrs.fr \\ Équipe Codisant \\ COgnition DIstribuée dans les Systèmes Artificiels et NaTurels \\ LabPsyLor (EA 3947) - Université Nancy 2 \\ Équipe Silex \\ Supporting Interaction and Learning by Experience \\ Liris (UMR 5205) - Université Lyon 1
}

\begin{abstract}
Tracking two co-users' activity: The case of a joint writing situation mediated by a numerical artefact. Our research aims at observing a joint and mediated writing situation. In the activity we studied, two users were asked to co-write a procedural text with the help of a numerical artefact, composed of three types of resources: a video showing the content to be described, a chat and a text editor. During this activity, numerical interaction tracks were rendered visible. Tracks concern interactions between users and interactions between users and resources. We qualified these tracks and their properties, which concerned either the user or the numerical resource "sparking off" tracks, or the use of tracks made by users. Then, we revealed how the observation of the mobilisation of these tracks by users allowed us to capture the development of activity and the appropriation of the numerical artefact by users. Finally we discussed possible future works, and we presented some design trails for "tracing systems".
\end{abstract}

KEYwords

Joint activity, experimental situation, mediation, activity development, numerical interaction tracks, numerical environment.

\section{1.- Introduction : Contexte de la recherche et positionnement du travail}

Le travail présenté dans cet article relève d'une thématique de recherche commune aux équipes Codisant ${ }^{1}$ et Silex $^{2}$ : les activités humaines conjointes supportées par des dispositifs techniques. L'équipe Codisant s'intéresse en particulier à la production conjointe d'artefacts comme étant une activité à la fois sociale, cognitive et instrumentale. L'équipe Silex se penche quant à elle sur les systèmes informatiques présentant des traces des interactions entre acteurs, et sur leur utilisation en situation d'apprentissage médié. Dans ces équipes, nous pensons que la question de l'appropriation par les utilisateurs des dispositifs techniques supportant l'activité est centrale, bien que peu de recherches étudient les éléments de la situation qui favorisent cette appropriation. En effet, les environnements informatiques, et en particulier ceux pour l'apprentissage humain, sont souvent complexes et rarement « intuitifs » malgré les efforts de leurs concepteurs. La question de leur appropriation et de leur « re-connaissance » en tant qu'instruments par les utilisateurs motive fondamentalement les recherches menées dans ces équipes.

Dans le cas d'une activité instrumentant les interactions, comme c'est le cas dans une situation d'uti-

1. COgnition DIstribuée dans les Systèmes Artificiels et NaTurels, http://www.univ-nancy2.fr/pers/brassac/Codisant/

2. Supporting Interaction and Learning by Experience, http://sliris.cnrs.fr/silex/ 
lisation d'un environnement informatique pour l'apprentissage humain, une partie de ces interactions devient tangible et susceptible d'être observée à la fois par l'humain et par l'environnement, les traces de ces interactions pouvant donc « naturellement» être révélatrices de cette appropriation. Certains artefacts numériques permettent de conserver les traces informatiques d'interactions tangibles pour un humain, et dans un format qui fait sens pour lui ${ }^{3}$ (utilisateur et/ou analyste). L'utilisation de ces traces à des fins d'analyse est assez répandue dans le domaine des activités médiées, tandis qu'elle est peu fréquente lorsque l'observateur en est le producteur lui-même. Ainsi, bien que les enjeux théoriques, en termes de connaissances sur l'activité humaine médiée par ordinateur et que les enjeux pratiques, en termes de conception d'environnements " centrée utilisateur », soient très importants, le champ de recherches sur la « ré-utilisation » de l'expérience sous forme de visualisation des traces d'interactions est un domaine de recherche peu exploré. L'étude de l'utilisation de ces traces informatiques d'interaction par les utilisateurs est au cœur des travaux de recherche de l'équipe Silex, qui considère que la visualisation des traces informatiques des interactions entre acteurs et artefact numérique peut jouer le rôle de « facilitateurs » d'appropriation de ces artefacts par les acteurs.

La recherche que nous présentons dans cet article relève de la psychologie clinique de l'activité, et constitue l'un des travaux fondamentaux de l'équipe Silex en ce qu'elle légitime l'orientation scientifique des travaux de l'équipe. Cette recherche vise en effet à étudier le rôle et le statut des traces informatiques d'interactions dans une activité humaine, conjointe, médiée et à distance.

C'est précisément une situation d'utilisation de traces informatiques d'interactions, par l'acteur à l'origine des traces, qui nous intéresse dans le travail présenté ici. Nous voulons observer si lors d'une tâche médiée de co-rédaction, les acteurs utilisent les traces informatiques d'interactions (traces apparaissant de facto à l'interface) pour leur activité. Si c'est le cas, nous voulons étudier la participation de cette utilisation au phénomène d'appropriation de l'artefact numérique par les acteurs, et au développement de l'activité. Pour cela, et en vue de comprendre quel peut être le rôle des traces d'interactions en situations médiées, nous réalisons une étude de cas d'activité conjointe médiée via un artefact numérique composé de différents espaces numériques (Leplat, 2002). Nous étudions cette activité, mise en situation, selon une approche interactionniste de la cognition selon laquelle le sens d'une situation émerge des interactions entre utilisateurs et entre utilisateurs et environnement. Nous réalisons une étude ethnographique des interactions ayant lieu, entre acteurs et entre acteurs et espaces numériques, et du rôle des traces dans les interactions. L'ensemble de ce travail se positionne selon une posture non mentaliste de la cognition, qui donne une grande place à la mobilisation des artefacts dans les processus cognitifs. Cette perspective rassemble Suchman et Hutchins, et soutient que la cognition humaine est située (Suchman, 1987) dans la matérialité de l'environnement et distribuée (Hutchins, 1995) entre les acteurs de la situation (Brassac, Fixmer, Mondada, \& Vinck, 2007).

Nous présentons premièrement dans cet article les objectifs de notre étude expérimentale ainsi que l'activité observée, une rédaction conjointe et médiée. Nous exposons ensuite les choix méthodologiques que nous avons faits pour l'observation de la mise en situation, qui relèvent d'une approche psycho-ergonomique d'inspiration ethnographique. Le détail de la mise en situation est alors donné: principes, préparation, déroulement de la session, artefact numérique et espaces à disposition des acteurs, et données produites. Puis nous expliquons la méthodologie que nous avons adoptée pour le traitement des données en justifiant les choix de montage vidéo et de transcriptions que nous avons faits. Nous présentons ensuite l'analyse d'une session d'activité conjointe et médiée. Cette analyse met en évidence des résultats intermédiaires, qui sont des propriétés des traces informatiques d'interactions et de leur utilisation au fil de la session. Nous révélons par la suite d'autres résultats sur le rôle et le statut des traces informatiques d'interactions dans le déroulement de l'activité. Un exemple d'analyse, par le commentaire d'un fragment d'extrait de la session, est alors donné à lire, puis nous présentons les résultats d'analyses menées sur les trois extraits. Une discussion des résultats considère ensuite les limites de cette étude et la conclusion vient apporter quelques pistes de conception pour la création de « systèmes traçants » supportant des activités conjointes.

3. Contrairement aux fichiers logs, traces des interactions utilisateur-système que la plupart des systèmes produisent 


\section{2.- Traces informatiques d'interactions et processus cognitifs en activité conjointe: Objectifs de l'étude expérimentale et activité observée}

Nous montrons, dans (Ollagnier-Beldame \& Mille, 2007), qu'il existe différents niveaux d'abstraction des informations tracées par les artefacts numériques, et que ce niveau varie selon l'usage qui est fait de ces traces. Les traces informatiques d'interactions peuvent en particulier être présentées à l'acteur dans leur forme «brute ». Nous pensons aux traces d'interactions qui, dans certains environnements, se trouvent de facto présentes à l'interface. C'est le cas de certaines interfaces pour les activités conjointes et en particulier de certaines interfaces communicationnelles, comme les éditeurs de textes collectifs ou les chats où l'acteur voit constamment à l'écran les traces «brutes » de ce qu'il a fait précédemment, ainsi que les traces des actions des autres acteurs puisqu'il s'agit dans ce cas d'activités conjointes. Il est important de remarquer ici que l'on peut donc parler de traces informatiques d'interactions pour désigner des « empreintes » de l'activité des acteurs inscrites par et dans l'environnement, alors que l'environnement n'a pas été développé pour cela. Ce sont précisément à ces traces « brutes » que nous nous intéressons dans l'étude expérimentale présentée ici, celles qui apparaissent de facto à l'interface de certaines applications d'activité conjointe.

Nous croyons que la présentation de ces traces aux acteurs peut favoriser leur appropriation des artefacts numériques. Selon Millerand, Giroux et Proulx (2001), l'appropriation ne peut être comprise que dans le cadre d'un processus temporel impliquant des transformations de la situation par les utilisateurs. Ainsi dans cette perspective, l'utilisateur choisit (ou redéfinit) les fonctionnalités du dispositif pour donner un sens à son usage jusqu'à ce que celui-ci fasse l'objet d'une nouvelle définition. Et les « détournements d'usage » observés peuvent alors être compris comme des « révélateurs des dynamiques d'appropriation différenciée à l'œuvre dans la formation des pratiques ». Selon ces auteurs, c'est « dans » l'expérience de l'utilisateur avec le dispositif que l'appropriation se joue, c'est dans la « mise en objet» de la technique. Pour Rabardel (1995), l'appropriation résulte d'un processus progressif de genèse instrumentale, processus qui n'est pas linéaire, qui est difficile, et qui engendre des tensions entre l'objet de l'appropriation et l'usage fait de cet objet dans un contexte particulier. Nous retenons cette définition de Rabardel, et rajoutons que pour nous, l'appropriation est un processus itératif où la négociation de sens qui se joue dans l'utilisation d'un objet par un individu autorise l'adoption et l'adaptation de l'objet. C'est en quelque sorte l'action de donner du sens à une situation « qui n'en a pas ». Ce processus d'appropriation se situe selon nous entre deux moments de stabilisations de sens, dans des périodes « intermédiaires », et conduit à une transformation de l'individu en même temps que la transformation de la situation qui s'opère par cet « ajout de sens ».

Pour cette étude, nous nous sommes appuyés sur le cadre de la cognition située et distribuée pour mener une mise en situation, avec les attentes suivantes. Premièrement, nous voulons caractériser le rôle et le statut des traces d'interactions entre acteurs et entre acteurs et artefact numérique dans le processus d'appropriation de l'artefact par les acteurs. Deuxièmement, nous souhaitons exploiter nos observations sur l'utilisation des traces dans l'activité, pour mettre en évidence les propriétés de l'activité concernant la distribution entre les acteurs et les propriétés d'ancrage dans la matérialité.

Nous présentons dans une première partie les objectifs de notre étude expérimentale, et l'activité que nous observons, une activité de rédaction conjointe médiée par un artefact numérique. Il s'agit d'une activité de co-conception, au sens premier du terme, c'est-à-dire qu'il s'agit d'une activité de production conjointe de forme, ici discursive. Ce type d'activité est fréquemment proposé à des acteurs en situation mobilisant un artefact numérique pour l'apprentissage humain. Dans cette étude, les acteurs n'accomplissent pas explicitement une tâche d'apprentissage mais nous pensons que l'activité qu'ils effectuent est source d'un développement individuel. Le développement auquel nous référons est, selon Vygotski, lié à l'apprentissage par une relation d'unité (mais non d'identité) et réfère au processus par lequel un individu connait des transformations et des changements au cours du temps. Il s'agit du déploiement, de l'engendrement des phénomènes cognitifs survenant dans le 
rapport qu'un individu entretient au monde et par lesquels ce faisant et se faisant, il se transforme. Il n'est pas limité au développement chez l'enfant, mais concerne l'individu tout au long de sa vie, et c'est un processus dont la durée peut être de quelques minutes à plusieurs années. Dans son ouvrage «Mind in society. The development of higher psychological process », Vygotski décrit cette « temporalité micro » du développement: «Any psychological process (...) is a process undergoing changes right before one's eyes. The development in question can be limited to only a few seconds, or even fractions of seconds. » (Vygotski, 1978, p. 61). Ainsi, nous pensons que dans l'œuvre de Vygotski, l'adjectif « développemental » peut être compris comme synonyme de « processuel».

\section{1- Objectifs de l'étude expérimentale}

Cette étude expérimentale vise à répondre aux deux attentes exposées ci-dessus qui peuvent être opérationnalisées de cette manière: Premièrement, nous voulons observer l'utilisation des traces d'interactions comme « mine d'expériences » (opérations de retours ascenseurs, de copier/coller, etc. et éventuelle évolution de ces opérations) dans le processus d'appropriation de l'artefact numérique, et préciser le rôle et le statut de ces traces. Deuxièmement, nous voulons interroger la distribution de l'activité entre co-rédacteurs et rendre compte des caractères situés (ancré dans les « espaces informatiques »), distribués (entre les deux co-rédacteurs) et opportunistes (par l'interprétation indexicale des évènements) de la co-rédaction, médiée et à distance du mode d'emploi.

Nous supposons que la visualisation de traces de l'activité à travers l'histoire interactionnelle est source d'un développement potentiel des acteurs, au sens de Vygotski. Notre objet de recherche ne peut être appréhendé que par la mise en place de situations, avec une certaine validité écologique, et non par la manipulation de variables. Ainsi, nous avons mis en place une expérimentation au sens ethnographique du terme, qui met en situation une co-rédaction d'un mode d'emploi par deux acteurs à distance, via un artefact numérique. Nous présentons cette activité ci-dessous et la manière dont nous l'avons mise en situation pour l'étudier.

\section{2- Activité observée : une rédaction conjointe et médiée}

L'activité que nous observons est une rédaction conjointe et médiée d'un texte procédural. Bon nombre de recherches (Kraut, Egido, \& Galegher, 1990; Dillon, 1993 ; Mitchell, Posner, \& Baecker, 1995 ; Cerratto, 1999; Cerratto, \& Rodriguez, 2002; Cerratto Pargman, 2005) ont étudié la manière dont les personnes écrivent ensemble. La plupart de ces travaux s'accordent sur le fait que l'écriture collaborative implique des moments d'écriture et des moments de communication, des périodes d'activité synchrone où le groupe travaille en même temps et des périodes de travail seul, où les membres du groupe travaillent de manière asynchrone. Dans cette activité, il est parfois difficile d'identifier les productions de l'activité de co-rédaction et de distinguer s'il s'agit du texte à co-écrire ou de productions à visée communicative. En fait, chaque co-auteur avance dans la production du texte commun, basé sur sa perception des actions des autres. La tâche que nous proposons ici se rapproche de ce que Dausendschön-Gay et Krafft (1999) appellent les « rédactions conversationnelles » qui sont des situations où deux ou plusieurs personnes, le « système écrivant », se mettent autour d'une table pour concevoir et rédiger un texte commun. Dans ce type d'activité, les chercheurs ont montré qu'il y a d'abord une étape de construction de l'espace interactionnel qui s'opère, pendant laquelle les acteurs identifient et délimitent l'espace et le temps du travail collectif. En parallèle, le rapport social, les rôles dans l'interaction et la réalisation de la tâche se mettent en place via l'activité finalisée et les interactions. De notre point de vue, le couplage des cognitions individuées aux mondes social et matériel est central. Comme processus intersubjectif, cette négociation de sens revêt une dimension conjointe et une dimension individuelle (Brassac, \& Grégori, 2003). En effet, l'acteur interagit avec son partenaire de co-rédaction par et avec les espaces numériques à sa disposition pour l'activité (Rabardel 1995). Il y a négociation conjointe de sens, avec l'autre acteur, pour la production du mode d'emploi. Mais il y a aussi négociation réflexive de sens, de nature meta, au fil du déroulement de l'activité. Dans cette recherche, nous étudions cette activité lors d'une mise en situation 
et cherchons à observer les actions, discursives et non-discursives, que les acteurs mettent en œuvre pour réaliser l'activité conjointe. Nous adoptons une approche interactionniste et constructiviste pour analyser le canevas fait de ces actions, à l'origine de l'activité des acteurs. Ainsi, nous cherchons à étudier en profondeur l'apparition de certains instants de l'activité et la mobilisation de certains espaces, comme signes de progression ou d'étapes de l'activité. Pour nous, et selon Brassac (2001), le processus de rédaction conjointe est un processus cognitif collectif, c'est un « cas particulier de conception distribuée d'une inscription ». Il s'agit d'une activité intersubjective, car les actions du rédacteur, même s'il conçoit " en privé », sont adressées à d'autres: commanditaires, destinataires, co-rédacteurs, acteurs (y compris lui-même), etc.

Dans cette situation, les acteurs doivent rédiger un mode d'emploi décrivant comment réaliser un pliage origami de boîte en papier. Ce mode d'emploi est destiné à un adulte, que nous appelons le « tiers adressé ». Nous avons choisi la production de ce type de texte car il est de nature procédurale, complexe, et sans « traduction » textuelle unique. Cette production de texte n'est pas un problème à résoudre avec une solution unique. Par ailleurs, cette activité peut être réalisée par des adultes sans compétences professionnelles particulières. Les acteurs ont à rédiger de manière conjointe le mode d'emploi, en binôme, par le biais d'un artefact numérique constitué de deux ordinateurs en réseau et de ressources numériques. Ils sont à distance et ne se connaissent pas. Pour mener à bien leur travail, les co-rédacteurs se servent de ressources : une vidéo où l'on voit des mains réalisant le pliage qu'ils ont à décrire, un chat pour communiquer avec l'autre et un éditeur de textes pour produire le mode d'emploi. Le chat et l'éditeur de textes que nous utilisons sont des composants de Drew, développé à l'École des mines de Saint-Étienne (Corbel, Jaillon, Serpaggi, Baker, Quignard, Lund, \& Séjourné, 2003). Cet outil a été développé pour proposer différents modules de support à l'argumentation, dont le chat et l'éditeur de textes. Notre choix a été motivé par le fait que Drew possède un " rejoueur » d'interactions post-activité, qui produit des traces sans interprétation, mais exportées dans un tableur pour l'analyse de la situation a posteriori.

\section{3.- Méthodologie pour l'observation}

\section{1- Principes}

Nous étudions une activité médiée par le langage et par l'artefact numérique à la disposition des acteurs. Les acteurs communiquent et réalisent l'activité par le biais du clavier, de l'écran et de la souris de l'ordinateur en réseau qu'ils ont à disposition. Ils utilisent aussi la consigne papier qui leur est donnée en début d'activité. L'analyse de ce type de situation d'activité conjointe peut se baser sur un ensemble de documents permettant de rendre compte de plusieurs aspects de la situation de recherche: les modes d'emploi finalisés rendus par les co-rédacteurs, les traces d'activité ancrées dans le support numérique, les enregistrements vidéos des interactions (films des acteurs et des écrans), les réponses aux entretiens et aux questionnaires transcrits. Ces documents, principaux matériaux de nos analyses, sont des données construites en cours de mise en situation. Ces « construits 》 se rapprochent de ce que Latour nomme « obtenues $»^{4}$ (Latour, 2001, p. 49), par leurs caractères non pré-existant et émergeant à partir de la situation.

Pour notre recherche, nous avons choisi d'adopter une approche ethnographique pour étudier les interactions enregistrées sous forme de films et de traces, et pour interpréter ces interactions qui concernent la production du mode d'emploi via des (re)mobilisations d'expériences et des négociations de sens. Selon nous, seule une méthode qualitative et capable de révéler des processus interactionnels de grain très fin entre humains et entre humain et artefact numérique peut convenir ici; pour observer et analyser des processus aussi peu connus que ceux de l'appropriation de l'environnement via les traces informatiques d'interaction. Dans le cadre de notre mise en situation, l'activité des ac-

4. Latour, dans L'espoir de Pandore (2001, p.49) « Décidément, on ne devrait jamais parler de « données » mais toujours d' »obtenues »" 
teurs est enregistrée. Tous les acteurs ont leurs actions à l'écran (jeux de la vidéo origami et actions discursives : chat et éditeur de textes Drew) enregistrées par un logiciel de capture d'écran. Leurs actions discursives sont par ailleurs tracées par Drew lui-même. Certains acteurs sont également filmés en plan large, de manière à voir leur visage et leurs mains sur les périphériques.

\section{2.- Étude expérimentale : Mise en situation}

La mise en situation a eu lieu en février 2006 dans les locaux de l'Icom ${ }^{5}$ de l'Université Lyon2. Nous présentons ci-dessous l'objectif de cette mise en situation, la préparation qu'elle a nécessitée, son déroulement, l'environnement utilisé et les données qu'elle a permis de capter. L'ensemble des documents du plan de l'étude est largement détaillé dans (Ollagnier-Beldame, 2006).

\subsection{1- Acteurs}

Pour notre étude de cas, et compte tenu des choix méthodologiques exposés ci-dessus, un seul couple d'acteurs était suffisant. Cependant, par " précaution », nous avons décidé de convoquer quatre binômes pour réaliser l'activité afin d'augmenter nos chances d'avoir un lot complet de données pour les analyses, la machinerie installée pour notre mise en situation (ordinateurs, caméras vidéo, logiciel jouant de la vidéo origami et logiciel Drew) pouvant être à l'origine de « bogues ».

Ainsi, huit acteurs ont réalisé l'activité, tous étudiants en premier cycle à l'Université en filière « Information Communication ». Ils étaient tous familiers de l'utilisation d'un ordinateur en réseau, et en particulier du chat. Les acteurs ont travaillé en binôme, sans connaître leur partenaire de rédaction, pour éviter toute gêne dans leur comportement ou leur expression. Ces quatre binômes ont été filmés en plan large puis interrogés à l'issue de l'activité. Nous avons installé sur les machines des acteurs un logiciel pour générer et éditer des vidéos de sessions d'écran qui enregistre en continu les interactions à l'interface. Six des huit acteurs ont été filmés en plan large. Après une démonstration des outils (vidéo origami et différents espaces de Drew), les acteurs se sont répartis en deux salles (binômes « séparés »). Ils ont réalisé en cinq minutes une « micro-tâche » de co-rédaction, faisant intervenir les mêmes ressources que l'activité principale (éditeur de textes et chat Drew). Ils ont ensuite eu à réaliser l'activité de rédaction du mode d'emploi pour réaliser un pliage de boîte, en cinquantecinq minutes (binômes différents de la « micro-tâche »). À l'issue de l'activité, nous avons demandé aux acteurs de répondre à des questions par écrit, concernant en particulier les potentiels retours arrière qu'ils auraient pu faire (utilisation de l'« historique » du chat ou re-visualisation du contenu de l'éditeur de textes). Ensuite nous nous sommes entretenus de manière individuelle avec les acteurs qui étaient filmés pour leur demander une nouvelle fois de s'exprimer sur des éventuels « retours arrière » qu'ils auraient pu faire. Ces questions avaient pour objectif de revenir sur les points qui concernent nos attentes, c'est-à-dire l'utilisation de l'expérience passée en cours d'activité.

\subsection{2- Artefact numérique}

L'artefact numérique à disposition d'une paire d'acteurs se compose de deux ordinateurs connectés en réseau. Concrètement, chacun des deux acteurs est placé devant son ordinateur, lui-même relié à celui de son partenaire par internet. Au fur et à mesure de leurs frappes au clavier apparaissent donc des segments discursifs; ce, dans trois zones discursives bien différenciées à l'écran. Par ailleurs, une quatrième zone leur donne à voir le film du pliage; film qu'ils peuvent manipuler (retour arrière, arrêt sur image, etc.) à loisir. Les acteurs ne disposent par ailleurs d'aucune autre ressource matérielle. Pour réaliser cette tâche, chaque acteur dispose ainsi de quatre espaces à l'écran de son ordinateur, organisés selon l'interface suivante:

5. Institut de la COMmunication 


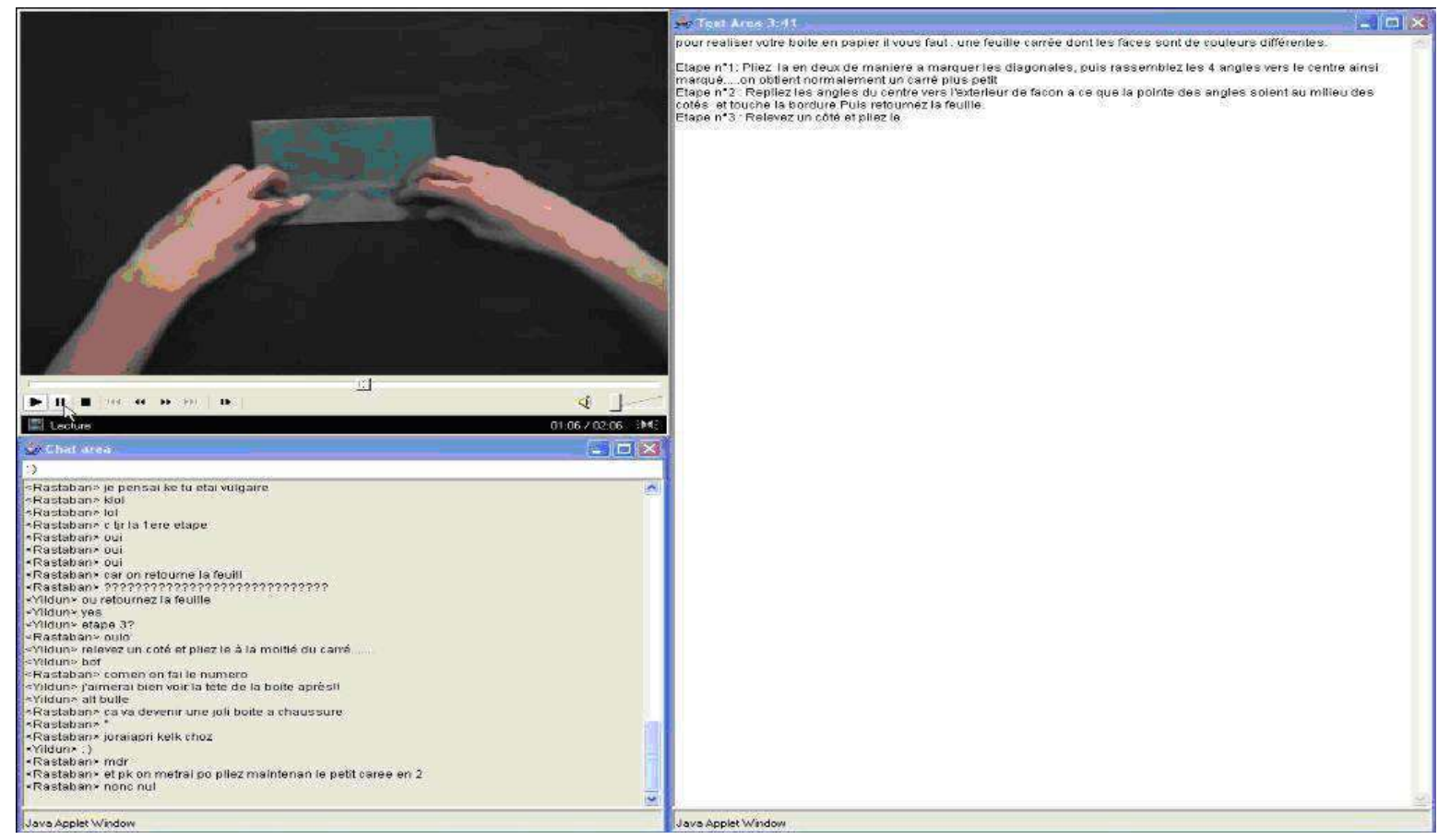

Figure 1: Interface en quatre espaces pour la mise en situation principale

Figure 1: Four-space interface for the main experimental situation

- La vidéo donnant à voir le pliage qui est un espace privé. Elle peut être jouée, pausée, accélérée, ralentie ou stoppée à souhait par les acteurs. Elle dure environ deux minutes.

- Le chat du logiciel Drew, développé pour tracer les interactions, qui est composé de:

- Un espace privé (zone de saisie), que nous appelons « chat privé » (car il n'est vu que par le rédacteur). Les acteurs y écrivent, puis publient en appuyant sur la touche « entrée ${ }^{6}$.

- Un espace partagé (zone de publication), que nous appelons « chat publié », visible par les deux acteurs.

- L'éditeur de textes qui est un espace partagé du logiciel Drew, amélioré pour notre expérimentation par Dyke (2006) pour avoir deux curseurs au lieu d'un seul. Ainsi, les deux acteurs y ont leur curseur et il est possible qu'ils y écrivent en même temps.

\subsection{3.- Données de la mise en situation}

Pour étudier l'activité des acteurs, nous retenons celles qui nous permettent de saisir le processus de co-rédaction médiée. Ce sont les données qui rendent compte de la transformation de l'activité au cours du temps, et non celles qui montrent seulement le produit de l'activité. Il s'agit des films d'écran et des traces des interactions via Drew, qui constituent la matière première principale de nos analyses. L'ensemble des données de la mise en situation se trouve dans (Ollagnier-Beldame, 2006).

Voici sur la figure 2 un exemple de traces que Drew génère, selon Dyke (2006).

6. Ainsi, lorsqu'un rédacteur construit son énoncé, il a le loisir d'aller et venir dans sa phrase, l'effaçant à rebours pour ensuite la réécrire, à l'identique ou non. Il doit ensuite réaliser une action de validation pour publier l'énoncé ainsi finalement élaboré. Ce faisant, il « sait » ce qu'il va donner à lire à son partenaire, il peut « contrôler » si ce qu'il adresse à l'autre contient bien ce qu'il intentionne de lui signifier. La captation que nous avons mise en place permet de visualiser, avec une granularité très fine (l'écriture d'un caractère), cette " micro-histoire » de la production d'énoncé dans la zone de chat privé. 


\begin{tabular}{|c|c|c|c|}
\hline & Speaker & Utterance & Tool \\
\hline $0: 07: 00$ & Yildun & $\begin{array}{c}\text { j'essaie sur l'editeur, un truc genre: vous } \\
\text { disposez d'une feuille carrée }\end{array}$ & chat \\
\hline $0: 07: 16$ & Rastaban & oui c porfait & chat \\
\hline $0: 07: 49$ & Rastaban & *begins writing** & textboard \\
\hline $0: 07: 49$ & Yildun & chat \\
\hline $0: 07: 50$ & Rastaban & $\begin{array}{c}\text { 1> vous } * * * \text { production : speaker was } \\
\text { interrupted }\end{array}$ & textboard \\
\hline $0: 07: 54$ & Yildun & oui vo mieu & textboard \\
\hline $0: 08: 07$ & Rastaban & chat \\
\hline $0: 08: 07$ & Yildun & $\begin{array}{c}1<\text { vous } 1>\text { vous disposez d'une feuille de } \\
\text { papier carrée } * * * \text { production }: \text { timeout }\end{array}$ & textboard \\
\hline
\end{tabular}

Figure 2: Trace informatique générée par le logiciel Drew Figure 2: Numerical track generated by Drew software

On trouve le temps en première colonne, le nom de l'acteur dans la deuxième, l'énoncé dans la troisième colonne, et le nom de l'outil utilisé pour l'action dans la quatrième colonne. Pour la colonne " énoncé », "**begins writing** » indique qu'un acteur positionne son curseur dans l'éditeur de textes, « $1>$ » indique que l'action d'écriture est à la ligne 1 et le « > » indique que l'acteur commence à écrire à cet endroit de la ligne. Après le « $1>»$, on a la production de l'acteur ajoutée à ce qu'il y a déjà dans l'éditeur le cas échéant. «*** production: speaker was interrupted » indique que l'acteur a été « interrompu » dans sa saisie par l'autre acteur, « $1<»$ indique que l'action d'écriture est à la ligne 1 et le «<» indique qu'il y avait déjà un texte à cette ligne, «*** production: timeout » indique que l'acteur a « interrompu » sa saisie car son curseur n'a pas bougé depuis plus de 5 secondes, ou bien parce qu'il a positionné son curseur ailleurs que dans l'éditeur de textes.

Ainsi dans l'exemple suivant (Figure 3), la ligne de trace Drew doit être comprise comme suit: à huit minutes et sept secondes, Yildun agit dans l'éditeur de textes. À la ligne 1 de l'éditeur, il y avait déjà d'inscrit « vous », elle rajoute « disposez d'une feuille de papier carrée », puis change d'espace numérique ou bien fait une pause longue de plus de 5 secondes.

\begin{tabular}{|l|l|l|l|}
\hline 0:08:07 & Yildun & $\begin{array}{l}1<\text { vous } 1>\text { vous disposez d'une feuille de } \\
\text { papier carrée *** production: timeout }\end{array}$ & textboard \\
\hline
\end{tabular}

Figure 3 : Extrait de la figure 2

Figure 3: Extract from figure 2

\section{4.- Méthodologie pour le traitement des données}

Pour mener nos analyses, l'activité d'un seul binôme a été retenue, de pseudonymes Rastaban et Yildun, deux jeunes femmes étudiantes à l'université Lyon 2. Nous explicitons ci-dessous les choix que nous avons faits sur la question de la mise en forme des données pour re-présenter les interactions actrice(s)-artefact numérique; ces choix méthodologiques concernant la lisibilité et la granularité des données que nous avons choisi de retenir pour nos analyses.

\section{1.- Montage en quadravision synchronisée des espaces discursifs}

Il a été nécessaire d'élaborer un corpus qui soit la pérennisation de l'événement labile qui s'est déroulé lors de l'expérimentation. Puisque les actrices n'ont pas parlé ni exprimé d'oralisations justifiant de conserver les films en plans larges pour le son, nous avons décidé de monter en quadravision synchronisée les seuls espaces discursifs des actrices (MQED): chats et éditeurs de textes, en y 
ajoutant les vidéos du pliage origami pour chaque actrice (Figure 4 ci-dessous) pour avoir les actions sur ces films de manière synchrone aux productions discursives. Nous avons réalisé ce travail avec un logiciel de montage vidéo.

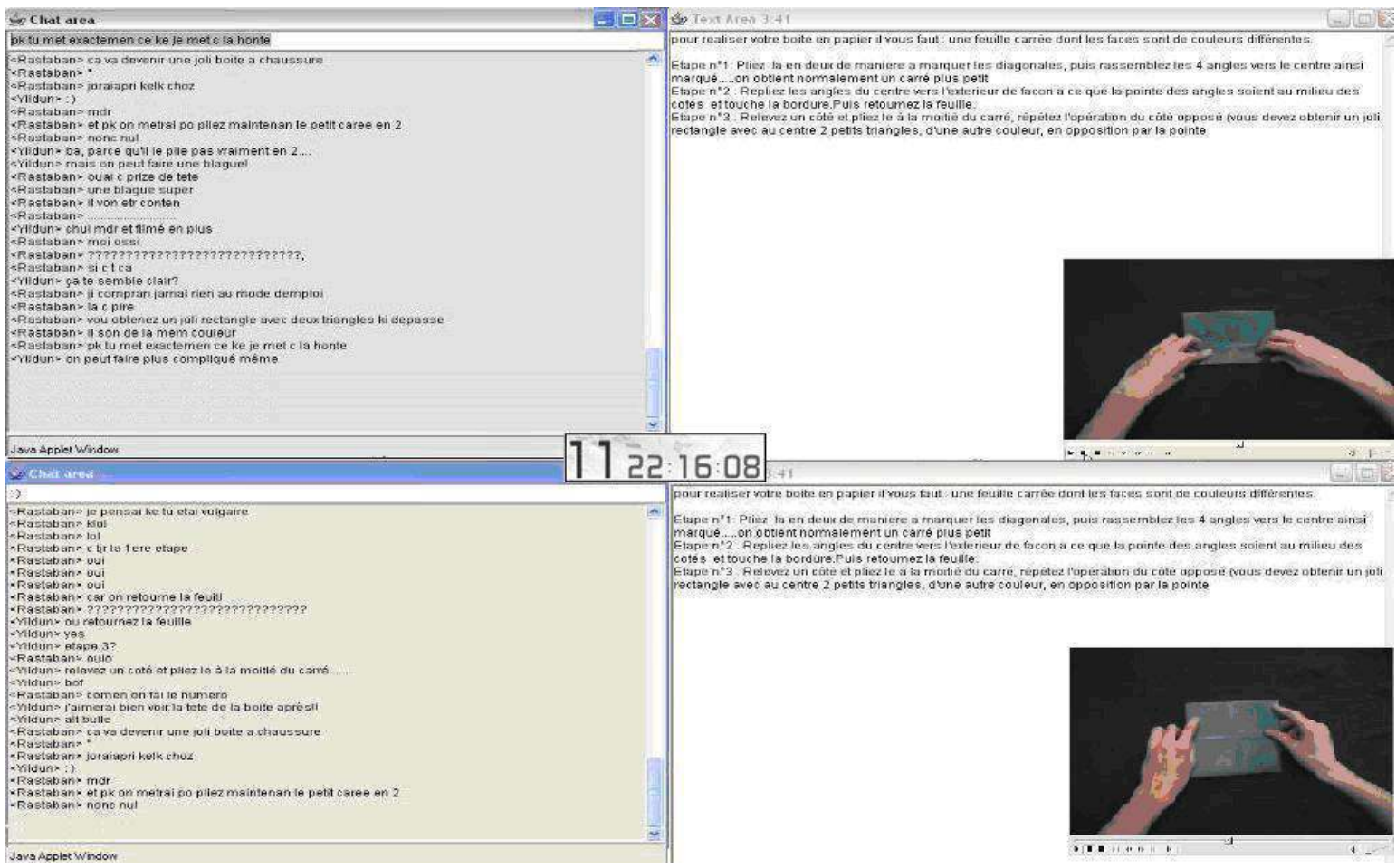

Figure 4: Montage en quadravision synchronisée des espaces discursifs et vidéo origami

Figure 4: Synchronised quadravision editing of discursive spaces and origami video

L'existence de trois zones de scription ne rend pas facile la transcription de ce corpus. Nous avons opté pour la solution suivante.

\section{2.- Méthode de transcription du corpus}

Nous avons réalisé une transcription des productions langagières de Rastaban et Yildun lors de l'activité, que nous présentons dans cette partie. Notre transcription apparaît sous forme de tableau, dans lequel nous avons fait cinq colonnes (voir Figure 5) : la première colonne indique le temps, la deuxième correspond au chat privé de Rastaban, la troisième est le chat publié, qui est l'espace du chat qui est conjoint, la quatrième colonne est le chat privé de Yildun, et la cinquième est l'éditeur de textes qui est aussi un espace conjoint. Chacune des quatre dernières colonnes comporte une sous-colonne de désignation des occurrences, et une sous-colonne de contenu des occurrences. Par occurrence, nous entendons un " accompli », la " réalisation d'une chose ». L'activité est un accomplissement continu de ces « réalisations de choses ». C'est également une suite d'entités. Par « entité », nous entendons une ou plusieurs productions porteuses d'un sens potentiel du point de vue de notre analyse a posteriori. Les entités sont constituées d'occurrences, qui peuvent être de différents types. Dans notre transcription, nous avons rapporté tout ce qui a été échangé sous forme discursive entre les actrices, selon des conventions de transcription détaillées dans (Ollagnier-Beldame, 2006). Nous présentons maintenant la méthodologie que nous retenons pour mener les analyses sur les données, qui s'appuie sur des principes ethnographiques. 


\section{5.- Analyse d'une session d'activité conjointe et médiée}

Rappelons que pour mener nos analyses, nous n'avons retenu que l'activité d'un seul binôme,

Rastaban et Yildun ${ }^{7}$. Pour l'analyse des interactions discursives, nous considérons que le statut d'un énoncé ne s'accomplit pas par l'usage même de la scription, mais qu'il faut tenir compte du processus par lequel il est perçu dans la rédaction conversationnelle, le sens de celle-ci se construisant par négociation entre les co-actrices, en émergeant des sens potentiels portés en chaque énoncé (Brassac, 2004).

\section{1.- Résultats intermédiaires : qualification des traces et de leur utilisation dans les extraits}

L'artefact numérique que les actrices utilisent leur fournit de facto des traces de leur activité. Elles les utilisent pour co-rédiger, et nous montrons ci-dessous en quoi ces traces et leur utilisation ont des propriétés spécifiques, en nous basant sur le montage MQED et la transcription des interactions que nous avons réalisés. Les propriétés que nous présentons ici sont considérées du point de vue des corédactrices. Il ne s'agit en effet pas de caractériser les traces de manière " absolue », mais de révéler leurs propriétés en prenant comme référentiel l'acteur qui les «produit » ou les utilise. Nous distinguons deux types de propriétés. Premièrement, nous identifions celles qui sont inhérentes aux traces des interactions, en lien avec la co-rédactrice qui est à l'origine des traces, ou en lien avec l'espace de l'environnement dans lequel elles apparaissent. Deuxièmement, nous présentons les propriétés qui sont en rapport avec l'utilisation qui est faite des traces par les co-rédactrices.

\subsection{1.- Qualification des traces selon l'acteur ou l'espace à l'origine des traces}

Selon l'acteur à l'origine des traces, et selon l'acteur qui les perçoit ou les utilise, les traces peuvent être de deux natures. Il peut en effet s'agir de traces dont l'acteur est « à l'origine », c'est-à-dire des inscriptions de ses interactions avec l'environnement, ou avec son partenaire via l'environnement. Dans ce cas nous appellerons ces traces des « traces propres ». Mais il peut également s'agir de traces dont le partenaire est à l'origine. Dans ce cas nous dirons que ce sont des « traces alter ».

Par ailleurs, selon l'espace numérique (chat privé, chat publié, éditeur de textes) dans lequel l'acteur laisse et peut percevoir les traces, nous identifions des propriétés différentes. Premièrement, nous proposons de considérer le chat privé et l'éditeur de textes comme étant des aires de « manipulation » et de potentielle « inscription » des énoncés, car les traces des productions y apparaissent dès que les productions sont saisies, et elles peuvent aussi être modifiées, voire disparaître (c'est systématiquement le cas des traces du chat privé, et cela peut l'être pour les traces de l'éditeur de textes). À l'opposé, nous considérons le chat publié comme étant « strictement» une aire d'inscription car dans cet espace les traces ne sont pas immédiates vis-à-vis des productions, ce sont des évènements et non des opérations c'est-à-dire qu'il s'agit de résultat d'opérations faites dans un autre espace. Ainsi ces espaces ne présentent pas la même labilité des traces qu'ils offrent. En effet, nous pouvons qualifier les traces du chat privé de " traces éphémères », car elles ne " durent » que le temps de la saisie de l'énoncé, avant publication ou effacement. Les traces de l'éditeur de textes sont quant à elles des « traces labiles », car elles sont sujettes à se transformer. En effet, l'éditeur de textes est un espace de manipulation et d'inscription, c'est-à-dire que les traces qu'il offre ne sont pas éphémères, elles peuvent durer, mais on peut aussi les modifier. Lorsqu'elles sont produites, elles ne sont pas inscrites « dans le dur» de l'espace. Enfin, les traces du chat publié sont quant à elles des « traces persistantes » car elles restent inscrites dans cet espace, sans aucune possibilité d'être modifiées. Deuxièmement, les traces des différents espaces ne sont pas toujours perceptibles par tous les acteurs. Nous abordons ici la propriété d'adressage de la trace, qui rend compte de la (ou des) actrice(s) pouvant

7. À titre indicatif, l'ampleur des échanges discursifs dans l'activité de ce binôme, ici scripturaux, se répartit comme suit: l'activité a duré 55 minutes. Environ 400 opérations ont été faites, dont environ la moitié d'opérations langagières, et la moitié d'autres opérations. Il y a eu 145 publications dans le chat publié, et le volume verbal du corpus est d'environ 1800 mots pour le binôme. 
la percevoir. Nous parlons bien ici de l'adressage des traces et non de l'adressage des énoncés produits. Il s'agit donc ici non pas d'un adressage intentionné des actrices, mais de la visibilité de facto des traces, pour l'une, l'autre ou les deux actrices. Nous avons identifié deux types d'adressage des traces. Premièrement, dans le chat privé, les traces sont adressées à l'actrice qui est en train d'écrire une production. En effet, elle est la seule à pouvoir percevoir la trace de ce qu'elle est en train de produire. Nous dirons qu'il s'agit de « traces auto-adressées ». Deuxièmement, nous avons un adressage similaire pour les traces du chat publié et celles de l'éditeur de textes. Dans le chat publié, la trace est principalement adressée à l'autre actrice (l'autre rédactrice), mais la productrice de cette trace est elle-même une adressée. Dans ce cas nous avons donc un adressage " double », et nous dirons qu'il s'agit de «traces hétéro-adressées ». Enfin dans l'éditeur de textes, la trace est destinée au binôme, c'est-à-dire adressée aux deux actrices. Nous dirons dans ce cas aussi qu'il s'agit de « traces hétéroadressées ».

\subsection{2.- Qualification de l'utilisation des traces}

Nous pouvons également déceler des propriétés des traces en fonction de l'utilisation qui en est faite par les co-rédactrices. Il s'agit dans ce cas davantage de caractéristiques de l'utilisation des traces plutôt que de propriétés qui leur sont propres. Nous avons repéré deux utilisations distinctes des traces dans la session que nous analysons. Premièrement, la (ou les) actrice(s) peu(ven)t « simplement » consulter les traces en cours d'activité. Il s'agit par exemple de lecture de l'historique du chat publié, que nous repérons en tant qu'analyste par des retours d'ascenseur dans cet espace. Dans ce cas, nous qualifions cette utilisation des traces de « consultatoire ». Deuxièmement, la (ou les) actrice(s) peu(ven)t effectuer des opérations sur les traces. Ces opérations peuvent être du copiage, du collage des traces. Dans ce cas, nous qualifions cette utilisation des traces d' « opératoire ».

Révélées par nos analyses, ces différentes propriétés des traces informatiques d'interaction et de leur utilisation constituent des indices, ressources sur lesquelles nous nous appuyons pour mettre en évidence les résultats suivants, qui concernent le rôle et le statut des traces informatiques dans le déroulement de l'activité observée.

\section{2.- Résultats consécutifs : rôle et statut des traces informatiques d'interactions dans le déroulement de l'activité}

La situation que nous avons étudiée est constituée de deux personnes en situation de rédaction conjointe, placées dans deux salles distinctes, face à un artefact numérique qui permet de communiquer par internet, via un chat. C'est une situation devenue très courante et qui donne lieu à des modes d'expression conversationnels nouveaux ${ }^{8}$. Les hésitations inhérentes à toute production verbale sont ici gommées par le filtre entre le « chat privé » et le « chat public ». Bref un grand nombre de phénomènes centraux dans la conversation classique sont ici absents. La question est de savoir ce que ce type de situation induit pour la construction de significations. Ceci a déjà été largement étudié mais pas dans cette situation où simultanément un espace d'écriture conjointe est présent sur chacun des deux écrans.

Dans cette étude, nous avons choisi trois extraits de la session d'activité que nous avons analysés (Ollagnier-Beldame, 2006). Choisir Le mode opératoire du choix de chaque extrait a été le suivant: nous avons constaté que, au fil de l'activité, la production du mode d'emploi, source de négociation conjointe de sens, se stabilise par « paliers ». Nous avons en effet remarqué des stabilisations à plusieurs moments dans la négociation du sens s'opérant au sein de l'activité de production. La fin de chaque extrait retenu est précisément marquée par des stabilisations de sens. Pour repérer le

8. Les acteurs doivent s'affranchir des modalités non verbales et para verbales. La production d'un énoncé n'est pas réalisée sous le regard de l'autre et le scripteur n'a pas la possibilité d'intégrer d'assentiment ou de désapprobation de l'autre. Le rire, non visible, doit être explicitement exprimé s'il veut être « dit». La demande d'avis ou de précision, le questionnement, l'étonnement doivent être précisément manifestés, voire explicitement mis en avant pour être perceptible par l'autre. 
commencement de l'extrait, nous avons « remonté » le fil des interactions jusqu'au moment où, selon nous, la négociation de sens s'oriente vers la stabilisation qui marque la fin de l'extrait. Le premier extrait montre l'accomplissement des préliminaires à la co-rédaction. Ce premier extrait présente une utilisation consultatoire de traces propres et alter du chat publié. Un deuxième extrait expose l'accomplissement de la rédaction conjointe de la phrase introductive du mode d'emploi, et illustre utilisation consultatoire de traces propres du chat publié et du chat privé. Troisièmement, nous avons repéré un extrait dans lequel nous voyons l'accomplissement de la rédaction conjointe de la première étape du mode d'emploi. Ce troisième extrait montre une utilisation opératoire de traces propres du chat publié. L'analyse complète de ces trois extraits peut être consultée dans (Ollagnier-Beldame, 2006). Nous présentons ci-dessous l'analyse d'un fragment du troisième extrait, comme exemple des analyses menées, puis nous exposons une synthèse des résultats des trois extraits analysés.

\subsection{1.- Exemple d'analyse : un fragment du troisième extrait}

Afin de montrer comment l'analyse des extraits s'est déroulée de manière concrète, nous présentons sur la figure 5 un fragment du troisième extrait d'une durée d'une minute et quarante-deux secondes. De ce fragment d'extrait, nous ne nous intéressons qu'à trois secondes en particulier (encadré noir), pendant lesquelles se produisent les opérations entourées en rouge sur la figure. Nous présentons cidessous l'analyse de ces trois secondes, à titre d'illustration de notre méthode.

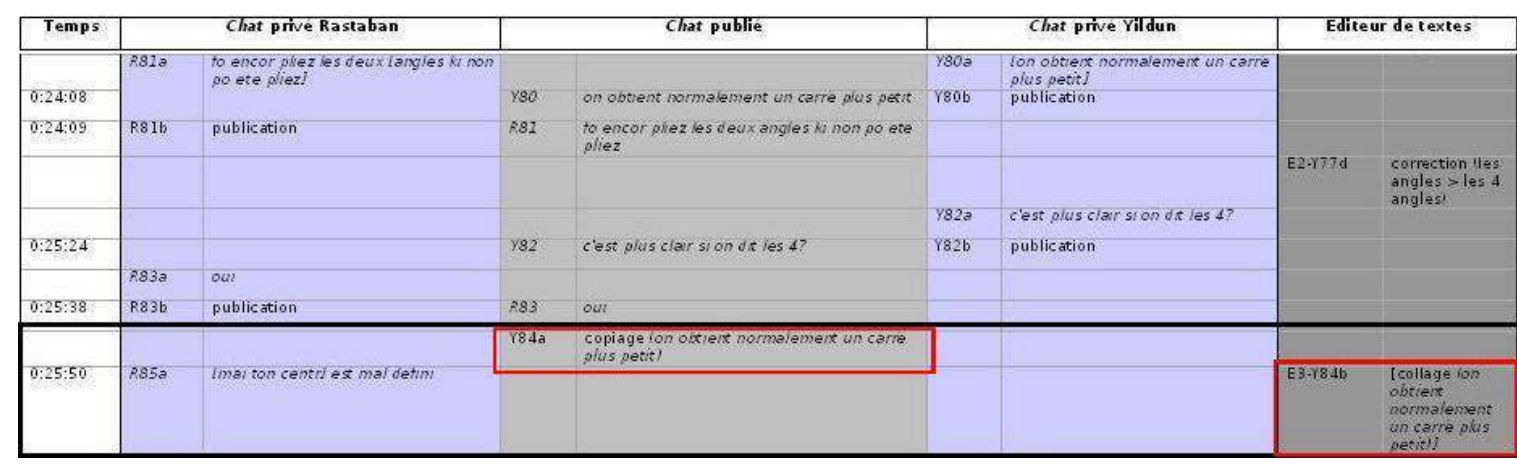

Figure 5: Fragment d'extrait analysé

Figure 5: Analyzed activity fragment

Si l'on veut « simplement » décrire ce qui se passe entre les deux co-rédactrices pendant ce fragment d'extrait de trois secondes, nous pouvons relater les éléments suivants: Yildun " revient » sur ce qu'elle avait publié dans le chat en Y80 avant que Rastaban ne formule une requête de modification en R81, et re-mobilise cette production qui lui est propre. Elle en copie l'inscription " on obtient normalement un carré plus petit » en Y84a, puis la colle ensuite à la troisième ligne de l'éditeur de textes en E3-Y84b.

L'analyse ethnographique plus complète des occurrences Y84a et E3-Y84b met quant à elle en évidence les éléments qui suivent: nous voyons que Yildun accomplit une utilisation opératoire d'une trace d'interaction " propre », qui nous intéresse pour plusieurs raisons. Premièrement, cette trace est une trace du chat publié, elle est donc principalement adressée à Rastaban, mais Yildun en est une adressée aussi (propriété d'adressage). Cette mobilisation de sa propre trace par Yildun (propriété d'appartenance) montre selon nous une utilisation « augmentée » de la trace, une répétition au-delà de la répétition. En effet, la première production en Y80 était une proposition formulée à Rastaban dans le chat pour une suite possible à donner au mode d'emploi, qui reste dans l'espace conversationnel. Mais au moment où Yildun la colle dans l'éditeur de textes, elle prend un statut plus finalisé, car elle se trouve directement notée dans l'espace d'inscription, qui est adressé au tiers. Pour le même contenu de la trace, nous voyons que le changement de son espace d'inscription entraine une transformation du statut de la trace dans l'activité. Nous supposons que cette trace est ainsi un intermédiaire à la conduite de l'activité, entre Yildun et l'environnement de l'activité. Cette intermédiation concerne 
selon nous le versant situé de l'activité cognitive de Yildun, c'est-à-dire son ancrage matériel dans le dispositif numérique. Enfin, par ce collage, la phrase « on obtient normalement un carré plus petit » est maintenant dans l'éditeur de textes, destinée au tiers adressé. C'est la première phrase du mode d'emploi qui contient un « on ». Est-ce parce qu'elle a été copiée depuis le chat et qu'elle n'était initialement pas destinée au tiers? Ce « on » sera-t-il modifié dans la suite de la construction du mode d'emploi ? Ce « on » marque-t-il un engagement des actrices qui se «mettent à la place » du tiers? Cache-t-il un « vous »? Nous ne le savons pas à cet instant de l'analyse. Seule l'analyse de la suite des interactions peut le dévoiler, cette analyse pouvant être lue dans (Ollagnier-Beldame, 2006).

\subsection{2.- Présentation des résultats des trois extraits analysés}

De l'analyse des trois extraits de la session d'activité de Rastaban et Yildun (Ollagnier-Beldame, 2006), nous obtenons des résultats qui viennent légitimer nos attentes concernant le rôle et le statut des traces informatiques d'interactions en tant que facilitateurs d'appropriation de l'artefact numérique par les actrices. Il n'est pas aisé de résumer ces résultats sans citer précisément les extraits et rapporter les interactions entre Rastaban et Yildun. Nous tentons cependant ci-dessous de rapporter les principaux résultats issus de nos analyses.

Nous soutenons que l'utilisation, au sein de l'interaction, des traces d'expérience supporte l'intersubjectivité et le rapport à l'artefact numérique. Nous affirmons que les mobilisations de l'expérience constituent des sources du processus de rédaction conjointe car elles configurent les modes d'expressions des individus. Observant que les expériences ne sont pas mobilisées de la même manière selon les moments de la session, nous avançons qu'à certains moments, des traces peuvent jouer un rôle de conjecture ou d'intermédiaire à l'activité. Nous supposons que via l'utilisation de traces, une prise de conscience de niveau meta est possible pour les actrices. Par ailleurs, au fil de la session, nous montrons que l'utilisation des traces évolue: au début de l'activité, les traces utilisées sont les traces propres, c'est-à-dire celles de ses propres productions. Les opérations de « retours ascenseur » que nous observons nous révèlent que leur utilisation est consultatoire, et concerne les traces du chat publié. Nous prétendons que ces traces prennent le statut d'objets intermédiaires pour l'activité, tour à tour dans les registres de la médiation, c'est-à-dire comme des objets communicationnels entre les actrices, et de la représentation, c'est-à-dire comme ponctuation de la rédaction du mode d'emploi. Nous voyons ensuite que les traces du chat privé sont utilisées, de manière consultatoire encore. Puis vers le milieu de la session, nous observons des opérations de « copiage-collage » et montrons une utilisation opératoire des traces propres du chat publié. Ce passage de l'utilisation consultatoire à l'utilisation opératoire est selon nous un signe de l'évolution de la genèse instrumentale de l'environnement. Il s'agit en particulier d'un moment d'instrumentalisation momentanée, processus micro dirigé vers l'artefact et à l'origine de l'enrichissement de ses propriétés, telle que le définit Rabardel « À un premier niveau, l'instrumentalisation est locale, liée à une action singulière et aux circonstances de son déroulement. L'artefact est instrumentalisé momentanément » (Rabardel 1995, p. 114). Dans la suite de la session, ce sont des traces des productions de l'autre qui sont mobilisées, de manière consultatoire. En fin d'activité, des traces alter sont utilisées de manière opératoire. Cette progression, des traces propres vers les traces alter, et de leur utilisation consultatoire vers leur utilisation opératoire, montre selon nous qu'il y a eu une actualisation des procédures d'utilisation des traces. Au plan micro, nous exposons comment les co-rédactrices sont parvenues de manière conjointe à produire une trace finalisée de leur activité, le mode d'emploi. Nous signalons l'importance de considérer un ou plusieurs « tours de scription » pour interpréter les énoncés et préciser leur rôle dans l'activité. Enfin, au plan macro, nous identifions plusieurs moments participant selon nous à la genèse instrumentale de l'artefact numérique par les co-rédactrices et de son évolution. Nous montrons que les traces ont un rôle dans ces genèses, et qu'ainsi, elles favorisent l'appropriation de l'environnement. Dans cette session d'activité, le mode d'emploi a acquis au fil de l'activité une forme stabilisée. Cette forme est le fruit du travail de deux rédactrices, qui ont été conduites, via une dynamique cognitive collaborative, à rédiger conjointement une production pérenne. Nous dévoilons comment la production des actrices s'est négociée et stabilisée dans le temps, par exemple via le jeu 
d'alternance dans l'utilisation des pronoms « on » et « je » et le rôle de ces pronoms. Nous exposons comment elle est devenue quasiment routinière en termes d'utilisation des espaces, et en parallèle à la prise en main des espaces numériques et à l'utilisation des traces d'expérience. Nous révélons également dans cette étude de cas le caractère opportuniste, situé et distribué de l'activité étudiée.

\section{6.- Discussion des résultats}

Dans ce travail, nous avons observé des acteurs utilisant un artefact numérique pour réaliser la tâche que nous leur avions assignée. À l'interface de leur environnement, des traces « brutes » de leur activité apparaissaient, au fil du déroulement de l'activité. Ces traces apparaissaient de fait, comme des « empreintes » de l'activité des acteurs inscrites par et dans l'environnement. Nous avons regardé si les acteurs utilisaient ou non ces traces. Nous avons observé qu'il y avait en effet des utilisations des traces, et que ces traces pouvaient être qualifiées différemment, selon l'acteur à l'origine des traces (et selon l'acteur qui les utilise), et selon l'espace numérique (chat privé, chat publié, éditeur de textes) dans lequel l'acteur laisse et peut percevoir les traces. Ces traces informatiques d'interaction se sont révélées être des objets supportant la négociation de l'activité, objets en continuelle évolution au cours du développement de l'activité.

D'un point de vue fondamental, cette étude nous a apporté des éléments de compréhension de la relation qui existe entre les traces informatiques d'interaction et les connaissances en co-construction: ces traces constituent des supports de communication entre les acteurs, et ce sont également des moyens d'interaction avec l'autre et avec soi-même, via une activité réflexive. D'un point de vue méthodologique, cette étude a montré qu'une investigation qualitative de type ethnographique permettait de mettre en évidence des processus micro ayant lieu entre les individus de la situation.

Bien entendu, cette mise en situation a présenté de nombreuses limites, en particulier au niveau de l'étendue des analyses tant au niveau du nombre de participants étudiés que du point de vue de la durée des extraits de la session considérés. Ce sont des occasions d'imaginer de nouvelles mises en situation qui viendront compléter les résultats présentés dans cet article, en particulier concernant la nature des propriétés des traces et de leur utilisation qui sont mises en évidence ici. Une des évolutions futures de ce travail est ainsi naturellement de consolider nos analyses selon ces deux limites, et en particulier la première. Notre objectif est de poursuivre les analyses de notre corpus, en leur donnant une épaisseur supplémentaire liée à l'interprétation de sessions d'autres binômes. Il est évident que les analyses que nous avons menées dans ce travail sont très coûteuses en temps, alors que nous n'avons analysé que quelques minutes d'une seule session d'activité. Il s'agira donc pour nous de trouver une démarche d'analyse qui allie la finesse d'interprétation à une réplicabilité des résultats entre binômes. Nous avons les données de trois autres binômes, et nous ne pourrons pas les analyser toutes avec la finesse des analyses réalisées ici. Mais nous pourrons en considérer une ou deux, par exemple en nous basant sur des réponses des participants aux questionnaires ou aux entretiens montrant des utilisations des traces d'interactions. Nous pourrons alors premièrement mettre en évidence des régularités dans l'utilisation des traces entre les binômes. Les données dont nous disposons sont également des traces de l'activité des binômes, mais cette fois qui nous sont destinées en tant qu' analyste de la situation. Il sera possible d'utiliser ces traces pour retrouver des « motifs » d'utilisation similaire des espaces puis de centrer notre étude sur les morceaux de corpus correspondant aux motifs. Deuxièmement, en vue d'évaluer si l'utilisation des traces d'interactions dans une activité conjointe et médiée est une activité largement située, distribuée et émergente, nous chercherons à voir si nous retrouvons les résultats obtenus dans cette recherche vis-à-vis des autres binômes. Nous envisageons de mettre au point une grille d'observation de l'activité, comportant les actions des participants le plus souvent rencontrées, et en particulier celles qui concernent l'utilisation des traces d'interactions. Cette grille pourra constituer un outil utile pour qualifier l'activité des binômes restants. Car même si nous perdrons beaucoup en finesse d'analyse, elle pourra être un moyen de repérer des motifs d'utilisation entre les binômes. Pour cette recherche de motifs, il sera intéressant 
d'utiliser un outil logiciel de codage de vidéos pour compléter le traitement de nos données et ainsi de pouvoir faire des analyses automatiques sur celles-ci, en particulier sur les propriétés des moments d'utilisation des traces.

\section{7.- Conclusion et perspectives : quelles recommandations pour la conception de « systèmes traçants " supportant des activités conjointes?}

Cet article a pris le parti de considérer les « traces informatiques d'interactions » comme des supports potentiels à la construction du sens telle qu'elle est mise en œuvre dans l'appropriation d'environnements informatiques, en particulier lorsque l'activité est conjointe.

Malgré les limites de nos résultats, quelles pistes de conception pouvons-nous en tirer pour la création de " systèmes traçants $»^{9}$ ? Ce type de systèmes, dans lesquels les interactions n'apparaissent pas de fait à l'interface, sont explicitement développés pour tracer les interactions entre acteurs et entre acteurs et environnement, et pour les présenter à l'interface. La littérature et l'expérimentation montrent bien que le potentiel d'utilisation est fort, mais montrent également les difficultés à offrir des moyens « appropriés » d'exploiter cette « mine » d'expériences (épisodes d'activité) à découvrir en cours d'activité, à réutiliser, à partager, à capitaliser, etc. Nous songeons principalement à la conception de systèmes qui renvoient à l'utilisateur en temps réel son histoire interactionnelle immédiate selon des « points de vue », ouvrant la voie à la prise de recul sur son activité et aux processus de développement humain associés. Selon nous il est très probable que le fait de rendre explicite à l'utilisateur son historique d'interactions avec le système, avec la possibilité d'agir sur cet historique, soit un élément à considérer comme un principe à suivre au sein du même processus de conception anthropocentrée des artefacts numériques, et en particulier de dispositifs pour l'apprentissage.

Il nous semble alors que la question principale est celle de la visualisation des traces informatiques d'interactions. En effet, le format de re-présentation des traces à l'utilisateur (en particulier sa «plasticité ») nous paraît être fondamental dans l'utilisation que les utilisateurs auront (ou non) de ces traces. Au sein de l'équipe Silex, nous réfléchissons au fait que l'environnement informatique « traçant » doit offrir des outils associés aux traces d'interactions, objets nouveaux à disposition de l'utilisateur pour son activité. Le concept de «transparence opérative » de Rabardel (1995, p. 150), qui désigne « les propriétés caractéristiques de l'instrument, pertinentes pour l'action de l'utilisateur, ainsi que la manière dont l'instrument les rend accessibles, compréhensibles, voire perceptibles pour l'utilisateur », doit selon nous modeler et figurer ces outils, et les propriétés de ces nouveaux objets « traces ». En particulier, un système traçant devrait selon nous mettre à disposition de l'utilisateur des moyens de se re-présenter ce qu'il est en train de faire par des interactions sur les traces informatiques d'interactions. Ceci suppose « d'inventer » les manières d'interagir avec des traces. Une piste est celle de la mise à disposition des utilisateurs de moyens de comparer leurs traces à différents temps d'activité ou aux traces d'autrui; ceci à des fins d'argumentation, de confrontation, de construction de sens commun, etc., toutes choses permettant de sensiblement enrichir les possibilités d'activités conjointes à distance.

Pour ouvrir plus largement le débat sur les usages possibles des traces d'interaction dans la conception et l'exploitation des environnements informatiques, et se rapprocher des préoccupations des situations d'apprentissage médiées, nous pensons qu'il est intéressant d'imaginer des perspectives permettant l'exportation des traces des utilisateurs vers les autres acteurs de l'apprentissage, une exportation supposant la capacité de transformer une trace " privée » en cours en " trace pour quelqu'un d'autre »; on peut imaginer alors des « reformulations » des « traces utilisateurs » (Cram,

9. Nous pensons aux systèmes informatiques qui enregistrent des séquences d'informations relatives à l'utilisation qu'un utilisateur en a fait et qui les mettent en forme « intelligibles » par l'humain (autres que des fichiers logs) pour les lui présenter, que ce soit l'utilisateur lui-même ou un analyste de la situation. On trouvera dans (Ollagnier-Beldame, 2006) une classification de ces « systèmes traçants » en quatre familles. 
Jouvin, \& Mille, 2007), ouvrant la voie à d'autres façons d'échanger sur les processus mobilisés dans l'activité en cours.

Enfin, concernant la mise en place de situations d'apprentissage médié, nous avançons que le choix de dispositifs numériques offrant de facto une visualisation des traces d'interaction peut permettre aux apprenants de prendre du recul quant à leur activité et d'avoir un meilleur contrôle de leur apprentissage en tant que processus situé dans le temps et dans les espaces numériques proposés. Nous pensons en effet qu'en contexte d'apprentissage, les situations d'activités conjointes ont réellement beaucoup à gagner à la mise en place d'environnement numériques permettant l'exploitation des traces informatiques d'interaction: en effet, dans de telles situations, la construction du sens est une nécessité explicite et nous imaginons que la négociation argumentative peut tirer grand profit d'un soutien par des traces explicites des interactions acteur-acteur et acteur-environnement informatique (Dillenbourg, 1999).

En conclusion, il nous semble que les perspectives de ce type de recherches, montrant les processus d'appropriation et d'utilisation des traces informatiques d'interactions par les acteurs, complétées par les recherches sur la mise en forme et la visualisation des traces, semblent pouvoir s'ouvrir vers une meilleure caractérisation des situations de régulation des situations d'activité conjointes et médiées, en particulier vers la compréhension des dynamiques d'échange entre acteurs.

\section{RÉFÉRENCEMENT}

Beldame, M. (2008). Suivre à la trace l'activité de deux co-acteurs: Le cas d'une rédaction conjointe médiée par un artefact numérique, @ctivités, 5 (1) pp. 40-57, http://www.activites.org/v5n1/v5n1.pdf

\section{RÉFÉRENCES}

Brassac, C. (2001). Rédaction coopérative: un phénomène de cognition située et distribuée. In M.-M. de Gaulmyn, R. Bouchard, \& A. Rabatel (Eds.), Le processus rédactionnel, écrire à plusieurs voix (pp. 171-193). Paris: L'Harmattan.

Brassac, C. (2004). Action située et distribuée et analyse du discours: quelques interrogations. Cahiers de Linguistique Française, 26, 251-268.

Brassac, C., Fixmer, P., Mondada, L., \& Vinck, D. (2007). Interweaving objects, gestures, and talk in context. Mind, Culture and Activity: An International Journal (sous presse).

Brassac, C., \& Grégori, N. (2003). Une étude clinique de la conception collaborative: la conception d'un artefact. Le Travail Humain, 66 (2), 101-127.

Cerratto, T. (1999). Instrumenting Collaborative Writing and its cognitive tools. In Proceedings HCP'99 Conference, Human Centred Processes, September 24-26. Brest, France, pp. 141-147.

Cerratto, T., \& Rodríguez, H. (2002). Studies of Computer Supported Collaborative Writing Implications for System Design. In M. Blay-Fornarino, A. Pinna-Dery, K. Schmidt, \& P. Zarat (Eds.), Proceeding of 5 th International Conference on the Design of Cooperative Systems, Saint-Raphael, France, 4-7 June (pp. 139- 154). Amsterdam: IOS Press.

Cerratto Pargman, T. (2005). Pour une conception des technologies centrée sur l'activité du sujet. Le cas de l'écriture de groupe avec collecticiel. In P. Rabardel, \& P. Pastré (Eds), Modèles du sujet pour la conception: Dialectiques activités développement (pp. 157-188). Toulouse: Octarès Editions.

Corbel A., Jaillon P., Serpaggi X., Baker M.J., Quignard M., Lund K., \& Séjourné A. (2003). DREW : Un outil Internet pour créer des situations d'apprentissage coopérant. In Actes de la conférence EIAH 2003, Strasbourg, pp. 109-113.

Cram, D., Jouvin, D,. \& Mille, A. (2007). Visualizing Interaction Traces to improve Reflexivity in Synchronous Collaborative e-Learning Activities. In The Proceedings of the 6th European Conference on e-Learning, Copenhague (in press). 
Dausendschön-Gay, U., \& Krafft, U. (1999). Environnement écrivant et processus de mises en mots dans les rédactions conversationnelles. Langages, 134, 51-67.

Dillenbourg, P. (1999). What do you mean by collaborative learning? In P. Dillenbourg (Ed.), Collaborativelearning: Cognitive and Computational Approaches (pp.1-19). Oxford: Elsevier.

Dillon, A. (1993). How collaborative is collaborative writing? An Analysis of the production of two technical reports. In M. Sharples (Ed.), Computer Supported Collaborative Writing (pp. 69-86). London: SpringerVerlag.

Dyke, G. (2006). Extension of the Musette framework for synchronous collective activities. Masters Thesis, EPFL, Lausanne, Switzerland.

Hutchins, E. (1995). Cognition in the Wild. Cambridge (MA): MIT Press.

Kraut, R., Egido, C \& Galegher, J. (1990). Patterns of communication in scientific research collaboration. In J. Galegher, R. Kraut, \& C. Egido (Eds.), Intellectual Teamwork. Hillsdale, N.J.: Lawrence Erlbaum Press.

Latour, B. (2001). L'espoir de Pandore. Pour une version réaliste de l'activité scientifique. Paris: Edition La découverte.

Leplat, J. (2002). De l'étude de cas à l'analyse de l'activité. Pistes, 4 (2), 1-31. http://pistes.uqam.ca/

Millerand, F., Giroux, L., \& Proulx, S. (2001). La « culture technique » dans l'appropriation cognitive des TIC. Une étude des usages du courrier électronique. In Actes du colloque international ICUST 2001, Paris, pp. 400-410.

Mitchell, A., Posner, I., \& Baecker, R. (1995). Learning to Write Together Using Groupware. In Proceedings of CHI'95, pp. 288-295.

Ollagnier-Beldame, M. (2006). Traces d'interactions et processus cognitifs en activité conjointe: Le cas d'une co-rédaction médiée par un artefact numérique. Thèse de Doctorat, Université Lumière Lyon2, France.

Ollagnier-Beldame, M., \& Mille, A. (2007). Faciliter l'appropriation des EIAH par les acteurs via les traces informatiques d'interactions? Rapport de recherche de l'équipe du laboratoire LIRIS.

Rabardel P., (1995). Les hommes et les technologies: Approche cognitive des instruments contemporains. Paris: Armand Colin.

Suchman, L. (1987). Plans and Situated Actions. Cambridge: Cambridge University Press.

Vygotski, L. (1978). Mind in society. The development of higher psychological process. Cambridge \& London: Harvard University Press.

\section{RÉSUMÉ}

La recherche présentée ici s'intéresse à une situation de rédaction conjointe et médiée, dans laquelle deux acteurs doivent co-rédiger un texte procédural à partir d'un artefact numérique constitué de trois types de ressources: une vidéo donnant à voir le contenu à décrire, un chat et un éditeur de textes. Lors de cette activité, des traces informatiques d'interactions entre acteurs et entre acteurs et espaces numériques apparaissent. Nous qualifions les propriétés de ces traces, qui relèvent soit de l'acteur ou de l'espace numérique qui en est «à l'origine », soit de l'utilisation qui en est faite par les acteurs. Nous dévoilons ensuite en quoi l'observation de la mobilisation de ces traces par les acteurs permet de suivre le développement de l'activité et l'appropriation de l'artefact numérique par les acteurs. Nous discutons enfin des perspectives de ce travail, et apportons quelques pistes de conception que nous pouvons tirer de nos résultats, pour la création de « systèmes traçants ».

\section{MoTS CLÉS}

Activité conjointe, artefact numérique, développement de l'activité, 
expérimentation, traces informatiques d'interaction.

\section{RESUMEN}

Seguir de cerca la actividad de dos co-autores: el caso de redacción conjunta mediada por un artefacto numérico. La investigación presentada aquí indaga una situación de redacción conjunta y mediada en la cual dos actores debes co-redactar un texto procedimental a partir de un artefacto numérico constituido por tres tipos de recursos: un video en el que se visualiza el contenido a describir, un chat y un ed itor de textos. Durante esta actividad, aparecen huellas informáticas entre actores y entre actores y espacios numéricos. Calificamos las propiedades de esas huellas que tienen que ver ya sea con el actor o el espacio numérico que las origina, o con la utilización que los actores hacen de ellas. Luego revelamos porqué la observación de la movilización de estas huellas por los actores permite supervisar el desarrollo de la actividad y la apropiación, por parte de los actores, del artefacto numérico. Finalmente, discutimos las perspectivas este trabajo y aportamos algunas pistas de diseño que podemos extraer de nuestros resultados, para la creación de « sistemas de trazabilidad ».

Palabras Clave

Actividad conjunta, artefacto numérico, desarrollo de la actividad, experimentación, huellas informáticas de interacción.

Article soumis le 22 juin 2007 accepté pour publication le 18 fevrier 2008 